\title{
Mass-Loss During the RR Lyrae Phase of the HB: Mass Dispersion on the HB and RR Lyrae Period Changes
}

\author{
Rebecca A. Koopmann, Young-Wook Lee, Pierre Demarque, and \\ Jamie M. Howard \\ Department of Astronomy, Yale University, New Haven, CT, USA
}

Horizontal branch (HB) models were evolved using the Yale stellar evolution code, YREC, to test the possibility that mass loss during the RR Lyrae phase is able to produce the observed color (mass) dispersion on the HB (Willson and Bowen 1984) and the anomalous period changes in RR Lyrae stars (Laskarides 1974). Models of total mass $0.64,0.66,0.68,0.70$, and $0.72 \mathrm{M}_{\odot}\left(\mathrm{Y}_{\mathrm{MS}}=0.23, \mathrm{Z}=0.001\right)$ were evolved with constant mass loss rates of $0,10^{-10}$, and $10^{-9} \mathrm{M}_{\odot} \mathrm{yr}^{-1}$. Mass loss was assumed to occur only in the RR Lyrae phase, and the instability strip was defined by $3.800<\log \mathrm{T}_{\text {eff }}<3.875$.

HB stars which lose mass evolve further to the blue. Low mass loss rates do not affect the shape of the tracks significantly. Stars, which without mass loss could not become blue HB stars, were able to emerge from the instability strip on the blue side. The trapping phenomenon predicted by Willson and Bowen (1984) was present in the case of the $0.68 \mathrm{M}_{\odot}$ star with a $10^{-9} \mathrm{M}_{\odot} \mathrm{yr}^{-1}$ mass loss rate. Trapping occurs when mass loss, which causes a star to move blueward, is balanced by the redward evolutionary trend. Synthetic HB's were calculated from the tracks with and without mass loss. The addition of mass loss does produce a slightly larger spread in mass, but these HB's do not resemble observed HB's (Koopmann et al. 1993, in preparation). We conclude that mass loss in the RR Lyrae phase cannot explain the dispersion in color on the HB.

Periods of RR Lyrae were computed using the period-density relation derived by van Albada \& Baker (1971). We find that the magnitude of period changes in stars without mass loss is similar to those with mass loss. Positive period changes have somewhat smaller magnitudes when mass loss is included. This was expected since the effect of mass loss is to impede the evolution to the red. Though mass loss in principle should increase the rate of evolution to the blue (and therefore increase the magnitude of negative period changes), the blueward portion of the evolution is so slow that mass loss rates tested here did not greatly speed the blueward evolution. We conclude that much larger rates of mass loss would be necessary to cause large negative period changes observed in some RR Lyrae stars.

In summary, mass loss on the HB of the magnitude tested here is not capable of explaining either the color dispersion in the $\mathrm{HB}$ or the large negative period changes in RR Lyrae stars. Larger mass loss rates would have an observable effect on HB morphology and are therefore unlikely.

Laskarides, P. G. 1974, Astrophys.\&Sp.Science, 27, 485

van Albada, T. S. \& Baker, N. 1971, Ap.J., 169, 311

Willson, L. A. \& Bowen, G. H. 1984, Nature, 312, 429 